\title{
Mesenchymal Stem Cells Attenuate Renal Fibrosis via Exosomes-Mediated Delivery of microRNA Let-7i-5p Antagomir
}

\author{
Juan Jin* \\ Fengmei Qian* \\ Danna Zheng \\ Wenfang $\mathrm{He}$ \\ Jianguang Gong \\ Qiang $\mathrm{He}$
}

Department of Nephrology, Zhejiang Provincial People's Hospital and Affiliated People's Hospital, Hangzhou Medical College, Hangzhou, Zhejiang, 3100I4,

People's Republic of China

*These authors contributed equally to this work
Correspondence: Jianguang Gong; Qiang $\mathrm{He}$

Department of Nephrology, Zhejiang Provincial People's Hospital and Affiliated People's Hospital, Hangzhou Medical

College, 158 Shangtang Road, Xiacheng

District, Hangzhou, Zhejiang, 3100|4,

People's Republic of China

Email gojigu3II@163.com;

qianghe1973@I26.com
Background: Renal fibrosis is a chronic and progressive process affecting kidneys in chronic kidney disease (CKD). Mesenchymal stem cells-derived exosomes (MSCs-Exo) have been shown to alleviate renal fibrosis and injury, but the mechanism of MSCs-Exoinduced renal protection remains unknown.

Methods: In this study, MSCs were transfected with let-7i-5p antagomir (anti-let-7i-5p), and then exosomes were isolated from the transfected MSCs to deliver anti-let-7i-5p oligonucleotides to inhibit the level of let-7i-5p in kidney tubular epithelial cells (NRK-52E).

Results: In both NRK-52E cells stimulated by TGF- $\beta 1$ and the mouse kidneys after unilateral ureteral obstruction (UUO), we demonstrated increased level of let-7i-5p. In addition, MSCs-Exo can deliver anti-let-7i-5p to reduce the level of let-7i-5p in NRK-52E cells and increase the expression of its target gene TSC1. Moreover, exosomal anti-let-7i-5p reduced extracellular matrix (ECM) deposition and attenuated epithelial-mesenchymal transition (EMT) process in transforming growth factor beta 1 (TGF- $\beta 1$ )-stimulated NRK-52E cells and in the kidneys of UUO-treated mice. Meanwhile, mice received exosomal anti-let $-7 \mathrm{i}-5 \mathrm{p}$ displayed reduced renal fibrosis and improved kidney function when challenged with UUO. Furthermore, exosomal anti-let-7i-5p promoted the activation the tuberous sclerosis complex subunit $1 /$ mammalian target of rapamycin (TSC1/mTOR) signaling pathway in vivo and in vitro.

Conclusion: In conclusion, exosomal anti-let-7i-5p from MSCs exerts anti-fibrotic effects in TGF- $\beta 1$-induced fibrogenic responses in NRK52E cells in vitro as well as in UUOinduced renal fibrosis model in vivo. These results provided a novel perspective on improving renal fibrosis by MSCs-Exo.

Keywords: chronic kidney disease, renal fibrosis, mesenchymal stem cells, exosomes and microRNAs

\section{Introduction}

Chronic kidney disease (CKD) is a significant public health concern that affects about ten percent of the global population. ${ }^{1} \mathrm{CKD}$ is characterized by the progressive loss of kidney function over time. ${ }^{2}$ In addition, the main causes of CKD are hypertension, diabetes, chronic pyelonephritis, and glomerular diseases (glomerulosclerosis and IgA nephropathy, diabetic nephropathy and lupus nephritis). ${ }^{3-5}$ Evidence has shown that patients with diabetes or hypertension should be screened on an annual basis for CKD. ${ }^{6}$ Glomerular filtration rate (GFR) is the best index for estimation of functioning renal mass. ${ }^{6} \mathrm{CKD}$ can be divided into five 
stages based on estimated GFR (eGFR) levels. ${ }^{7}$ The stages of CKD range from mild loss of kidney function in stage $1\left(\mathrm{eGFR}>90 \mathrm{~mL} / \mathrm{min} / 1.73 \mathrm{~m}^{2}\right)$ to complete kidney failure in stage $5\left(\mathrm{eGFR}<15 \mathrm{~mL} / \mathrm{min} / 1.73 \mathrm{~m}^{2}\right){ }^{2,8}$ Stage 5 of CKD is known as end-stage renal disease (ESRD), and patients with ESRD require dialysis or a kidney transplant to stay alive. ${ }^{7,9}$ Because CKD can progress to kidney failure (also called ESRD) and even death, early diagnosis of this disease is important for timely preventive and therapeutic interventions and preventing a time-dependent decline in eGFR. ${ }^{10,11}$

Renal fibrosis is a final common pathway in CKD. ${ }^{12}$ In addition, renal fibrosis is characterized by excessive deposition of extracellular matrix (ECM) in the tubulointerstitial, which ultimately leads to renal dysfunction. ${ }^{12,13}$ For this reason, assessment of renal fibrosis is frequently used for analysis of human kidney biopsies. ${ }^{14,15}$ The effective prevention and therapy of renal fibrosis are critical for the treatment of CKD. ${ }^{16}$ It is well known that the mTOR/ p70S6K signaling pathway is activated in renal fibrosis in CKD. ${ }^{17,18}$ Thus, inhibition of mTOR signaling may attenuate renal fibrosis in CKD. ${ }^{19}$

MSC-based therapy has emerged as a novel therapeutic strategy for treating kidney diseases via kidney tissue repair. $^{20,21}$ It has been shown that MSCs exhibit a therapeutic effect in vivo via a paracrine mechanisms or directly differentiating into different renal phenotypes such as mesangial cells and tubular epithelial cells. ${ }^{22-24}$ Subsequent studies showed that MSC-derived exosomes exert renal-protective effects by transferring the genetic information to target cells. ${ }^{25}$ Exosomes, nano-sized extracellular vesicles $(40-100 \mathrm{~nm})$, have been found to play important roles in cell-to-cell communication and tissue repair and regeneration. ${ }^{26}$ In addition, MSCderived exosomes carry complex cargo, including nucleic acids, mRNA, microRNAs (miRNAs), long non-coding RNAs (lncRNAs). ${ }^{26,27}$ Indeed, the delivery of exosomes derived from MSCs has been shown to alleviate renal fibrosis and function by delivery of miRNAs. ${ }^{28,29}$ Wang et al found that exosomal miRNA-let-7c-derived from MSCs could attenuate renal fibrosis in mice with UUO. $^{29}$ However, the biological role of MSC-derived exosomal miRNAs in renal fibrosis remains largely unclear.

In the present study, we found that MSCs-derived exosomal anti-let-7i-5p could attenuate the pro-fibrogenic response of NRK52E cells induced by TGF- $\beta 1$ in vitro and improved renal function and attenuated renal fibrosis in
UUO-induced renal fibrosis model in vivo. These data may provide a novel perspective on improving renal fibrosis by MSCs-Exo.

\section{Materials and Methods Identification of Differentially Expressed miRNAs (DEMs)}

The original files of two independent datasets (GSE51674 and GSE42648) were downloaded from Gene Expression Omnibus database (GEO, https://www.ncbi.nlm.nih.gov/ geo/).

GSE51674 dataset contains miRNAs profile of 16 kidney specimens ( 6 specimens from patients with type 2 diabetes, 6 specimens from patients with diabetic nephropathy and 4 specimens from normal kidney patients who underwent kidney biopsy). $R$ language was utilized to analyze miRNAs levels in kidney tissues from patients with low $(<2 \mathrm{~g} ; \mathrm{n}=6)$ or high urine protein excretion $(24 \mathrm{~h}-\mathrm{UP}>2 \mathrm{~g} \mathrm{n}=10) .{ }^{30} 24 \mathrm{~h}-\mathrm{UP}>2 \mathrm{~g}$ was categorized as having high degree of CKD. |log2 Fold Change (FC) $\mid>1.5$ and $\mathrm{P}$-value $<0.05$ were set as the cut-offs to screen DEMs.

GSE42648 dataset contains miRNAs profile of 18 kidney specimens from 8 patients with lupus nephritis. $\mathrm{R}$ language was utilized to analyze miRNAs levels from kidneys with low $(<4 ; \mathrm{n}=9$ ) or high chronicity index (CI $\geq 4 ; \mathrm{n}=9) .{ }^{31} \mid \log 2$ Fold Change (FC) $\mid>0.667$ and P-value $<0.05$ were set as the cut-offs to screen DEMs.

The overlapping DEMs from GSE51674 and GSE42648 datasets were identified using the Venn diagram.

\section{Cell Culture and Treatment}

Human bone marrow-derived MSCs and rat kidney tubular epithelial (NRK52E) cells were obtained from Type Culture Collection of the Chinese Academy of Sciences (Shanghai, China). Cells were maintained in Dulbecco's modified Eagle's medium (Thermo Fisher Scientific, Inc., Waltham, MA, USA) supplemented with $10 \%$ fetal bovine serum (Thermo Fisher Scientific) and $100 \mathrm{U} / \mathrm{mL}$ Penicillin/ Streptomycin at $37^{\circ} \mathrm{C}$ in a $5 \% \mathrm{CO}_{2}$ incubator. NRK52E cells were treated with TGF- $\beta 1(10 \mathrm{ng} / \mathrm{mL})$ for $48 \mathrm{~h}$ to mimic in vitro model of fibrosis as previously described. ${ }^{32}$

\section{Reverse Transcription-Quantitative Polymerase Chain Reaction (RT-qPCR)}

TRIpure Total RNA Extraction Reagent (ELK Biotechnology, Wuhan, China) was used to extract total 
RNA from cells. For cDNA synthesis, RNA samples were reverse transcribed by using an EntiLink ${ }^{\mathrm{TM}} 1$ st Strand cDNA Synthesis Kit (ELK Biotechnology). After that, qPCR was carried out using the EnTurbo ${ }^{\text {TM }}$ SYBR Green PCR SuperMix (ELK Biotechnology) on the StepOne ${ }^{\mathrm{TM}}$ Real-Time PCR System (Thermo Fisher Scientific). The thermocycling conditions were as follows: $95^{\circ} \mathrm{C}$ for $3 \mathrm{~min}$, followed by 40 cycles of $95^{\circ} \mathrm{C}$ for $10 \mathrm{sec}, 58^{\circ} \mathrm{C}$ for $30 \mathrm{sec}$ and $72^{\circ} \mathrm{C}$ for $30 \mathrm{sec}$. Expression of target genes $\left(2^{-\Delta \Delta \mathrm{Ct}}\right)$ was normalized against $\beta$-actin and U6. Primer sequences are listed in Table 1.

\section{Cell Transfection}

The let-7i-5p agomir and let-7i-5p antagomir (anti-let-7i$5 \mathrm{p})$ and miRNA negative control (NC) were synthesized by GenePharma Co., Ltd. (Shanghai, China). MSCs were transfected with let-7i-5p agomir, anti-let-7i-5p or NC by

Table I Primer Sequences

\begin{tabular}{|c|c|c|}
\hline Name & & Primer Sequences $\left(5^{\prime}-3^{\prime}\right)$ \\
\hline \multirow[t]{2}{*}{ mmu-U6 } & Forward & CTCGCTTCGGCAGCACAT \\
\hline & Reverse & AACGCTTCACGAATTTGCGT \\
\hline \multirow[t]{2}{*}{ mmu-miR-let-7i } & Forward & TGGGTGAGGTAGTAGTTTGTGC \\
\hline & Reverse & CTCAACTGGTGTCGTGGAGTC \\
\hline \multirow[t]{2}{*}{ rno-U6 } & Forward & CCTGCTTCGGCAGCACAT \\
\hline & Reverse & CTCAACTGGTGTCGTGGAGTC \\
\hline \multirow[t]{2}{*}{ rno-miR-let-7i } & Forward & TGGGTGAGGTAGTAGTTTGTGC \\
\hline & Reverse & CTCAACTGGTGTCGTGGAGTC \\
\hline \multirow[t]{2}{*}{ mmu- $\beta$-actin } & Forward & CTGAGAGGGAAATCGTGCGT \\
\hline & Reverse & CCACAGGATTCCATACCCAAGA \\
\hline \multirow[t]{2}{*}{ mmu-COLIAI } & Forward & CTGACTGGAAGAGCGGAGAG \\
\hline & Reverse & CGGCTGAGTAGGGAACACAC \\
\hline \multirow[t]{2}{*}{ mmu- $\alpha-S M A$} & Forward & GATAGAACACGGCATCATCACC \\
\hline & Reverse & CATAATCTGGGTCATTTTCTCCC \\
\hline \multirow[t]{2}{*}{ rno-GAPDH } & Forward & GCCAAGGTCATCCATGACAAC \\
\hline & Reverse & GTGGATGCAGGGATGATGTTC \\
\hline \multirow[t]{2}{*}{ rno-COLIAI } & Forward & CCGTGACCTCAAGATGTGCC \\
\hline & Reverse & GAACCTTCGCTTCCATACTCG \\
\hline \multirow[t]{2}{*}{ rno- $\alpha-S M A$} & Forward & AGCATCCGACCTTGCTAACG \\
\hline & Reverse & CCAGAGTCCAGCACAATACCAG \\
\hline \multirow[t]{2}{*}{ rno-TSCI } & Forward & GATGGCCGCCTACAGAAACTA \\
\hline & Reverse & СТСТАССТССАСАGCTGGCC \\
\hline
\end{tabular}

Abbreviations: $\alpha$-SMA, alpha-smooth muscle actin; COLIAI, collagen $|\alpha|$; mmu, Mus musculus; rno, Rattus norvegicus; TSCI, tuberous sclerosis complex subunit I. using Lipofectamine 2000 reagents (Thermo Fisher Scientific) according to the manufacturer's instructions.

\section{Exosome Collection and Detection}

MSCs were cultured in exosome-free media, and the conditioned media (CM) was collected from MSCs. After that, exosomes were isolated from MSCs-CM by using the GETTM Exosome Isolation Kit (GeneExosome technologies, Freehold, NJ, USA) according to the manufacturer's instructions.

The concentration of exosomal proteins were determined using the bicinchoninic acid (BCA) assay kit (Beyotime, Beijing, China). Later on, $10 \mu \mathrm{g}$ of exosomes were loaded onto copper grid for $5 \mathrm{~min}$, and then the filter paper was used to remove excess liquid. After that, the grid was stained with $2 \%$ phosphotungstic acid (Servicebio, Wuhan, China) for $3 \mathrm{~min}$ and examined using a HT7700 Transmission electron microscopy (TEM; Hitachi, Tokyo, Japan).

Next, particle number and particle size of exosomes were detected using the ZetaView ${ }^{\circledR}$ Nanoparticle Tracking Analysis (NTA) instrument (Particle Metrix, Meerbusch, Germany).

\section{Co-Culture System}

MSCs were plated on 12-well Transwell $^{\circledR}$ polyester permeable supports (Corning, New York, NY, USA). Cy3labeled let-7i-5p was transfected into MSCs. Then, transfected MSCs on permeable supports were received either media alone or media + GW4869 $(20 \mu \mathrm{M})$. In addition, NRK52E cells were grown on the lower chamber. After 24 $\mathrm{h}$ of incubation, images were captured using a confocal microscope (Zeiss, Jena, Germany).

\section{Western Blot Assay}

Total proteins were quantified using the BCA assay kit (Beyotime), and equal amounts of protein $(30 \mu \mathrm{g})$ were separated by $10 \%$ SDS-PAGE. After that, proteins were transferred onto the polyvinylidene fluoride (PVDF) membrane (Millipore, Billerica, MA, USA). After blocking with 5\% skim milk in tris-buffered saline with tween-20 (TBST) for $1 \mathrm{~h}$, the membranes were incubated with primary antibodies: collagen $1 \alpha 1$ (1:1000, Cell Signaling Technology), fibronectin (1:1000, Abcam), alpha-smooth muscle actin ( $\alpha$-SMA; 1:1000, Abcam), TSC1 (1:1000, Abcam), phosphorylated-mTOR complex 1 (p-mTORC1; 1:1000, Cell Signaling Technology), mTORC1 (1:1000, 
Cell Signaling Technology), phosphorylated-p70 ribosomal s6 kinase (p-p70S6K; 1:1000, Cell Signaling Technology), p70S6K (1:1000, Cell Signaling Technology), phosphorylated-eukaryotic translation initiation factor 4E-binding protein 1 (p-4E-BP1; 1:1000, Cell Signaling Technology), 4E-BP1 (1:1000, Cell Signaling Technology), $\beta$-actin $\left(1: 1000\right.$, Abcam) at $4^{\circ} \mathrm{C}$ overnight. Later on, the membranes were incubated with the horseradish peroxidase-conjugated secondary antibody for $1 \mathrm{~h}$, and then the protein bands were visualized using the enhanced chemiluminescence (ECL) reagents (Thermo Fisher Scientific).

\section{Dual-Luciferase Reporter Assay}

Fragment of TSC1 3' untranslated regions (3' UTRs) contained the sequence of let-7i-5p binding sites was inserted into the luciferase reporter vector pGL6-miR (Beyotime). After that, NRK52E cells were co-transfected with pGL6miR-TSC1 3'UTR sequence and either let-7i-5p agomir or NC using Lipofectamine 2000 reagents. After $48 \mathrm{~h}$ of transfection, firefly and renilla luciferase activities in cell lysates were detected using the Dual-luciferase Reporter Assay System (Beyotime).

\section{Animal Study}

Female C57BL/6 mice (8 weeks old, 20-25 g) were purchased from Beijing Vital River Laboratory Animal Technology Co., Ltd. (Beijing, China). Animals were kept under controlled environmental conditions (12 $\mathrm{h}$ dark/light cycles; temperature, $20-222^{\circ} \mathrm{C}$ humidity, 55 $\pm 5 \%$ ), and fed water and a standard rodent feed ad libitum. The UUO model was established as previously described. ${ }^{33}$ Briefly, mice were anesthetized, and then the left ureter was exposed. After that, the left ureter was double ligated with a silk suture permanently. Shamoperated mice, which underwent the same surgical intervention except for ureteric ligation, were used as sham control. All experiments were approved by the Institutional Ethical Committee of the Zhejiang Provincial People's Hospital in accordance with the National Institutes of Health Guide for the Care and Use of laboratory animals.

To investigate whether MSCs-Exo were efficient drug delivery vehicles, in vivo imaging system were performed. Firstly, $250 \mu \mathrm{L}$ of PKH67-labeled MSCs-Exo $(1 \mathrm{mg} / \mathrm{kg}$ ) were intravenously injected into mice underwent UUO or sham surgery. After that, mice were anesthetized (isoflurane: $3 \%$ induction and $1 \%$ maintenance) and the fluorescence images were observed in live animals using a fluorescence imaging system (Xenogen, Alameda, CA, USA) at 0,1 and 2 day after injection.

To investigate the effect of MSCs-derived exosomes, animals were randomly separated into four groups: Sham, $\mathrm{UUO}, \mathrm{UUO}+\mathrm{MSCs} / \mathrm{NC}$ exosomes, UUO + MSCs/anti-let $-7 \mathrm{i}$ exosomes groups. After that, mice were intravenously injected with MSCs/NC exosomes or MSCs/anti-let-7i exosomes $(50 \mu \mathrm{g})$ twice a week. ${ }^{34,35}$ Later on, animals were killed on days 28 , and then serum and kidney tissues were collected. The eGFR in mice was measured as previously described. ${ }^{36}$

\section{Enzyme-Linked Immunosorbent Assay (ELISA)}

The commercial ELISA kits (mouse BUN kit, mouse CR kit; Nanjing Jiancheng Bioengineering Institute, Nanjing, China) were used to detect the levels of blood urea nitrogen (BUN) and creatinine (CR) in serum of mice and levels of $\mathrm{CR}$ in urine of mice according to the manufacturer's instructions.

\section{Histology Analysis}

The kidney tissues were placed into 4\% paraformaldehyde, and then embedded in paraffin. After that, the tissues were cut into 5- $\mu \mathrm{m}$ transverse sections. Later on, the sections of kidney tissues were stained with hematoxylin and eosin (H\&E), and masson's trichrome staining. A fluorescence microscope (Olympus, Tokyo, Japan) was used to captured histological images.

\section{Immunohistochemistry (IHC) Assay}

After being deparaffinized at $65^{\circ} \mathrm{C}$ for $2 \mathrm{~h}$, the slides were cooled for $1 \mathrm{~h}$ at room temperature. After that, the slides were blocked with $10 \%$ goat serum at room temperature for $50 \mathrm{~min}$, and then incubated with primary antibodies: collagen $1 \alpha 1$ (Cell Signaling Technology, 1:200), fibronectin (Abcam, 1:500) and $\alpha$-SMA (Abcam, 1:200) overnight at $4^{\circ} \mathrm{C}$. Later on, the slides were incubated with corresponding secondary antibodies for $20 \mathrm{~min}$. A fluorescence microscope was used to observed histological images. Image-ProPlus software used to quantitatively analyze the expression of each protein.

\section{Statistical Analysis}

All statistical analyses were performed using GraphPad Prism software (version 7.0, La Jolla, CA, USA). For the 
comparison of two groups, unpaired Student's $t$-test was applied. One-way analysis of variance (ANOVA) and Tukey's tests were carried out for multiple group comparisons. All data are presented as mean \pm standard deviation (SD). Differences were considered statistically significant at $\mathrm{P}$ value $<0.05$. All data were repeated in triplicate.

\section{Results}

\section{Let-7i-5p Level in in vitro and in vivo Model of Renal Fibrosis}

To identify the DEMs in CKD, we analyzed the data from GSE51674 and GSE42648 datasets that downloaded from GEO database. Hierarchical clustering analysis indicated distinguishable miRNA expression profiles between kidney tissues from patients with mild and serious renal diseases. A total of 94 DEMs were identified from the GSE51674 dataset, and a total of 33 DEMs were identified from the GSE42648 dataset (Supplementary Figures 1 and 2). In addition, 123 DEMs in two microarray datasets were compared between each other, and then the Venn diagram was drawn, showing the existence of three intersection miRNAs (miR-186-5p, miR-150 and let-7i), which served as candidate DEMs (Figure 1A). MiR-186-5p and miR150 have been shown to be involved in the development of renal fibrosis. ${ }^{37,38}$ However, the role of let- $7 \mathrm{i}$ in renal fibrosis remains unclear. In this study, we found that the level of let-7i-5p, as well as the levels of markers of ECM deposition and myofibroblast formation collagen $1 \alpha 1$ and fibronectin were significantly increased in TGF- $\beta 1$-treated NRK52E cells (Figure 1B). Similarly, the levels of let-7i5 p, collagen $1 \alpha 1$ and fibronectin were markedly increased in the kidneys of UUO mice (Figure 1C). To sum up, the level of let-7i-5p was upregulated in fibrotic models in vitro and in vivo.

\section{Isolation and Characterization of Exosomes}

Accumulating evidences have shown that MSCs-Exo could attenuate renal fibrosis in CKD. ${ }^{29,39-41}$ Then, we purified the exosomes from the CM of MSCs. TEM and NTA analysis demonstrated that MSCs-Exo had typical saucer-like double-layer membrane structures with diameters of 50-150 nm (Figure 2A and B). In addition, the markers of exosomes, such as CD9 and CD81 were detected by Western blot. The data indicated that extracellular vesicles were positive for CD9 and CD81 (Figure 2C). Collectively, exosomes were successfully extracted from MSCs.

\section{Anti-Let-7i-5p Can Be Transferred from MSCs to NRK52E Cells via Exosomes}

We further assess whether anti-let-7i-5p can be transferred from MSCs to NRK52E cells via exosomes. As shown in Figure 3A, the level of let-7i-5p was notably downregulated in MSCs after transfection with anti-let-7i-5p (MSCs/anti-let-7i-5p). Meanwhile, the level of let-7i-5p was decreased in exosomes derived from the CM of MSCs that were transfected with anti-let-7i-5p (MSCs/ anti-let-7i-5p exosomes) (Figure 3B).

Next, NRK52E cells were indirectly co-cultured with MSCs/anti-let-7i-5p, and then RT-qPCR was used to detect the level of let-7i-5p in NRK52E cells. As indicated in
A

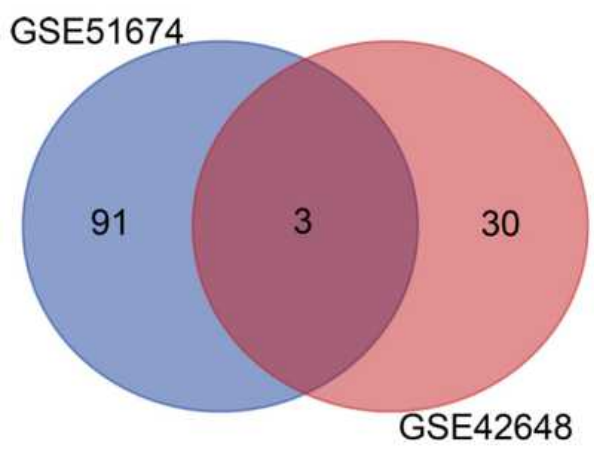

B

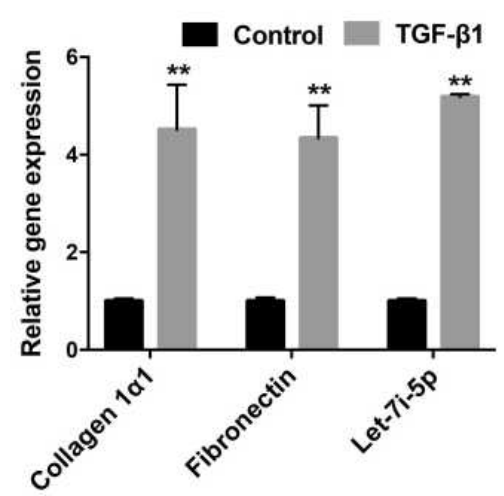

C

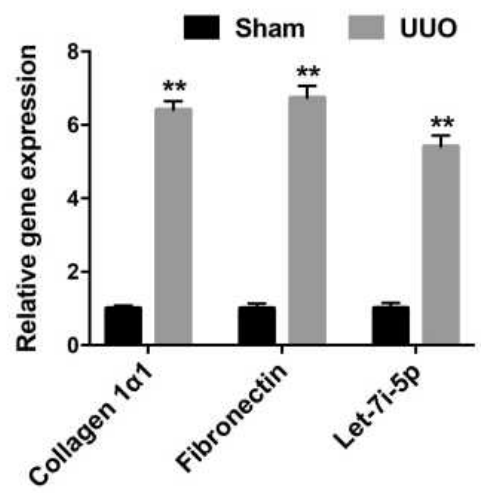

Figure I Let-7i-5p level in in vitro and in vivo model of renal fibrosis. (A) Overlapping DEMs were identified using R language. (B) NRK52E cells were treated with I0 ng/mL TGF- $\beta$ I. RT-qPCR was used to detect the level of let-7i-5p, collagen $|\alpha|$ and fibronectin in cells $(n=3)$. **P<0.0I compared with control group. (C) RT-qPCR was used to detect the level of let-7i-5p, collagen $|\alpha|$ and fibronectin in kidneys of UUO mice $(n=3)$. $* * P<0.01$ compared with sham group. The data were statistically analyzed using Student's $t$-test. 

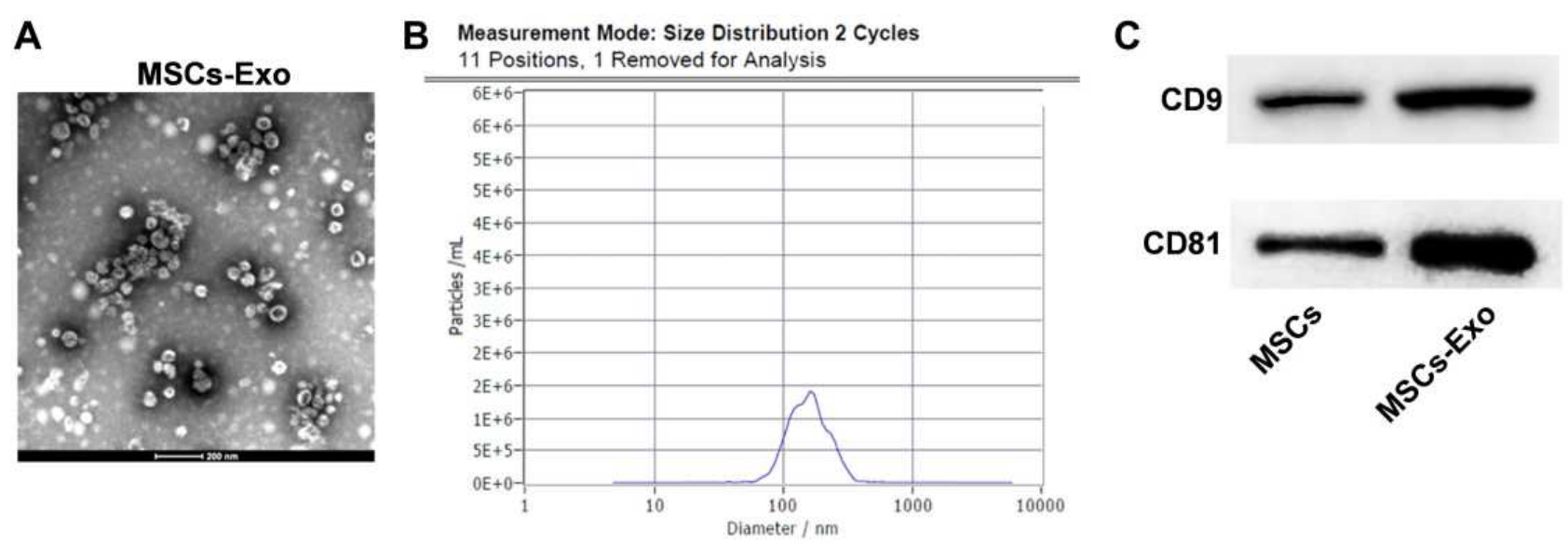

Figure 2 Isolation and characterization of exosomes. (A) TEM of exosomes derived from MSCs. (B) NTA was used to measure the size distribution of MSCs-Exo. (C) Western blotting analysis of CD9 and CD8I in MSCs and their exosomes $(n=3)$.

Figure 3C, let-7i-5p was significantly decreased in NRK52E cells co-cultured with MSCs/anti-let-7i-5p. Furthermore, NRK52E cells were indirectly co-cultured with MSCs that were transfected with cy3-labeled antilet-7i-5p. Cy3 fluorescence dye was observed in the incubated NRK52E cells (Figure 3D). However, with the treatment of exosome inhibitor GW4869, no cy3 fluorescence dye was detected in NRK52E cells (Figure 3D). Furthermore, the level of let-7i-5p in the CM of MSCs/ let-7i-5p were unchanged upon RNase treatment but notably decreased when treated with RNase plus Triton X-100 simultaneously, suggesting that extracellular let-7i-5p was mainly wrapped by membrane instead of being directly released (Supplementary Figure 3). Collectively, anti-let $-7 \mathrm{i}-5 \mathrm{p}$ can be transferred from MSCs to NRK52E cells via exosomes.

\section{MSCs-Secreted Anti-Let-7i-5p Inhibited the ECM Deposition and EMT Process in TGF- $\beta$ I-Treated NRK52E Cells}

To investigate the biological function of exosomal let-7i$5 \mathrm{p}$, the ECM deposition and EMT were detected in NRK52E cells with or without TGF- $\beta 1$. As shown in Figure 4A, NRK52E cells grown in DMEM medium with TGF- $\beta 1$ showed significantly increased expressions of collagen $1 \alpha 1$, fibronectin and $\alpha$-SMA compared with cells grown in DMEM medium without TGF- $\beta 1$; however, these TGF- $\beta 1$-induced changes were reversed when TGF$\beta 1$-treated NRK52E cells were indirectly cocultured with MSCs/NC or MSCs/anti-let-7i-5p. Consistently, both MSCs/NC exosomes and MSCs/anti-let-7i-5p exosomes markedly reduced the expressions of collagen III, fibronectin and $\alpha$-SMA in TGF- $\beta 1$-stimulated NRK52E cells (Figure 4B). These data suggest that MSCs-secreted let-7i$5 \mathrm{p}$ could inhibit the ECM deposition and EMT process in TGF- $\beta 1$-stimulated NRK52E cells.

\section{TSCI is a Functional Target of Let-7i-5p in NRK52E Cells}

To investigate how MSCs/anti-let-7i-5p exosomes could regulate ECM deposition and EMT process in NRK52E cells, four bioinformatics software tools (miRWalk, miRanda, RNA22 and Targetscan) were utilized to predict the potential binding targets of let-7i-5p. The data showed that TSC1 might be a potential target of let-7i-5p (Figure $5 \mathrm{~A})$. In addition, the luciferase activities of $3^{\prime}$ UTR of TSC1 were inhibited by let-7i-5p agomir (Figure 5B). Moreover, MSCs/anti-let-7i-5p exosomes notably increased the level of TSC1 in NRK52E cells (Figure $5 \mathrm{C})$. These data indicated that TSC1 is a binding target of let-7i-5p in NRK52E cells.

Furthermore, Western blot assay showed that TGF- $\beta 1$ resulted in decreased expression of TSC1 and increased phosphorylation of mTORC1, p70S6K and 4E-BP1 in NRK52E cells; however, these TGF- $\beta 1$-induced changes were reversed by MSCs/anti-let-7i-5p exosomes (Figure $5 \mathrm{D}$ and E). To sum up, MSCs/anti-let-7i-5p exosomes inhibited the ECM deposition and EMT process in TGF$\beta 1$-treated NRK52E cells via upregulation of TSC1.

\section{In vivo Distribution of MSCs/Exo}

Next, to assess whether MSCs/Exo can be efficiently delivered into the obstructed kidneys in UUO mice, the 

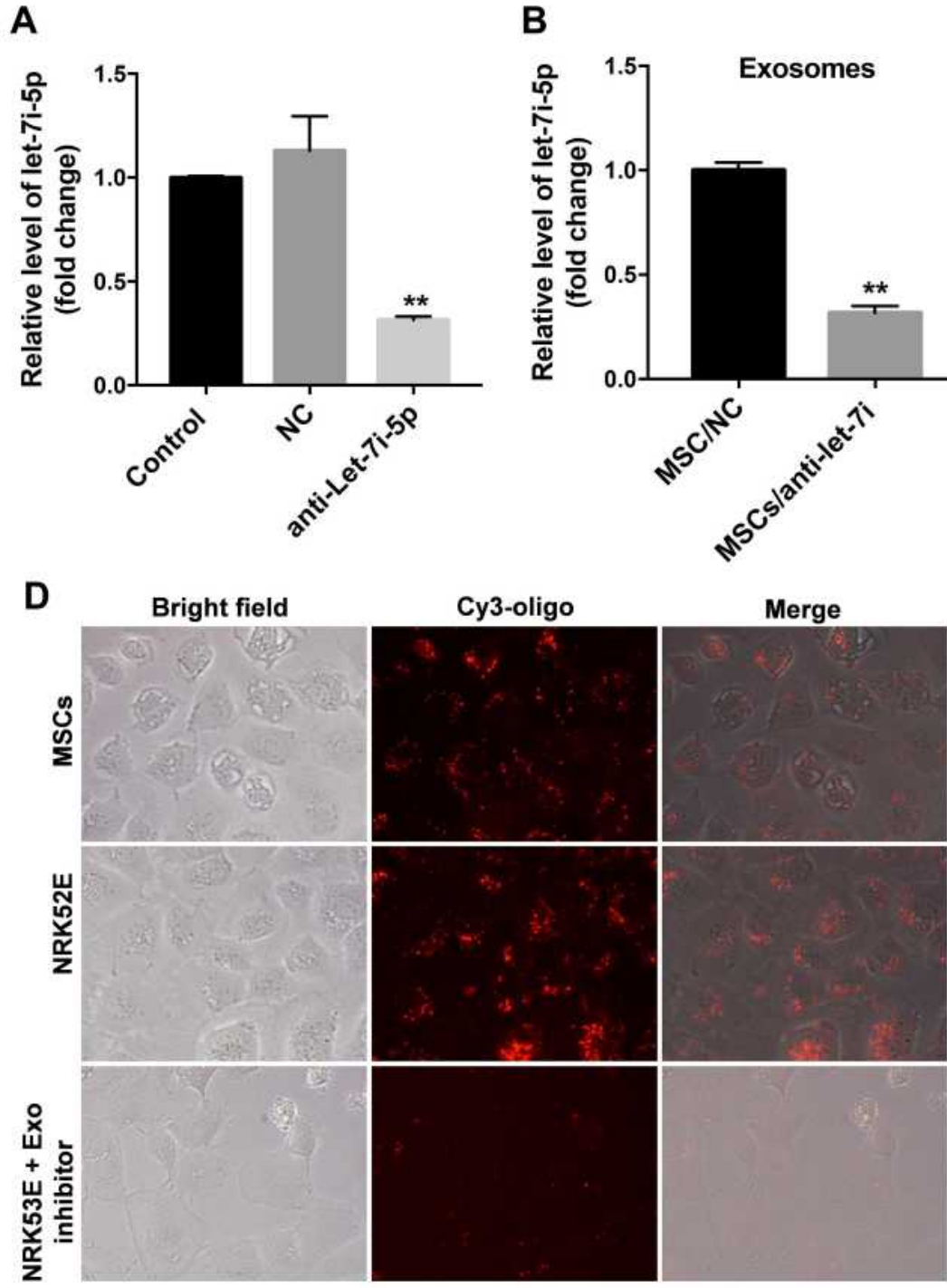

B

C
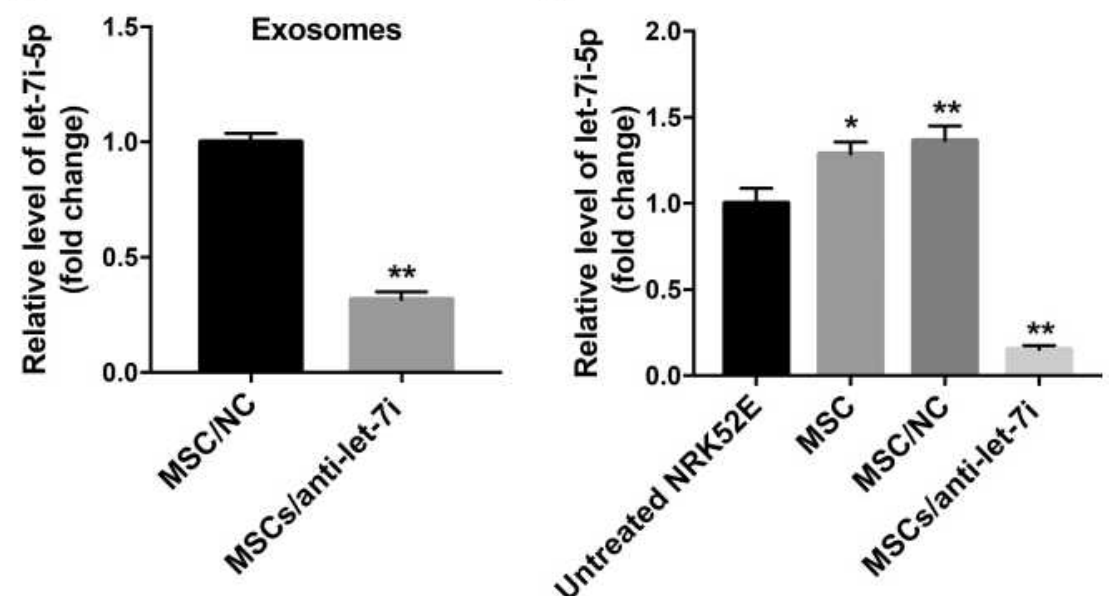

Figure 3 Anti-let-7i-5p can be transferred from MSCs to NRK52E cells via exosomes. (A) RT-qPCR was used to detect the level of let-7i-5p in MSCs transfected with let-7i$5 p$ antagomir $(n=3)$. **P < 0.0I, compared with NC group. (B) RT-qPCR was used to detect the level of let-7i-5p in MSCs/anti-let-7i-5p exosomes or MSCs/NC exosomes $(n=3)$. $* * P<0.01$, compared with MSC/NC group. (C) NRK52E cells were indirectly co-cultured with MSCs/anti-let-7i-5p or MSCs/NC. RT-qPCR was used to detect the level of let-7i-5p in cells $(n=3)$. $* \mathrm{P}<0.05$, **P $<0.01$, compared with untreated NRE52E group. (D) NRK52E cells were co-cultured with MSCs transfected with cy3-tagged let-7i-5p or GW4869-treated transfected MSCs. Fluorescence microscopy was used to observe exosomal transfer of let-7i-5p to NRK52E cells. The significance between two or more groups was analyzed by Student's $t$-test or one-way ANOVA respectively.

in vivo imaging system was performed. For tracking of MSC/Exo in vivo, PKH67 dye-labeled MSC/Exo were injected via tail vein into sham-operated mice or UUO mice. The results showed that MSCs/Exo mainly homed to the UUO injured kidney (Figure 6), indicating that MSCs-Exo could be served as a drug delivery carrier.

\section{MSCs/Anti-Let-7i-5p Exosomes Alleviated Renal Fibrosis in UUO Mice}

We further investigated the role of MSCs/anti-let-7i-5p exosomes in UUO-induced kidney fibrosis. As indicated in Figure 7A and B, UUO mice displayed impaired renal function, as determined by elevated serum BUN, serum $\mathrm{CR}$ and urine CR levels and reduced eGFR levels; however, these changes were reversed by both $\mathrm{MSCs} / \mathrm{NC}$ exosomes and MSCs/anti-let-7i-5p exosomes. In addition, mice subjected to UUO displayed inflammatory cell infiltration and interstitial expansion in kidney tissues; however, that effect was reversed by MSCs/anti-let -7i-5p exosomes (Figure 7C). Moreover, masson's trichrome staining showed that both MSCs/NC exosomes and MSCs/anti-let-7i-5p exosomes obviously decreased 


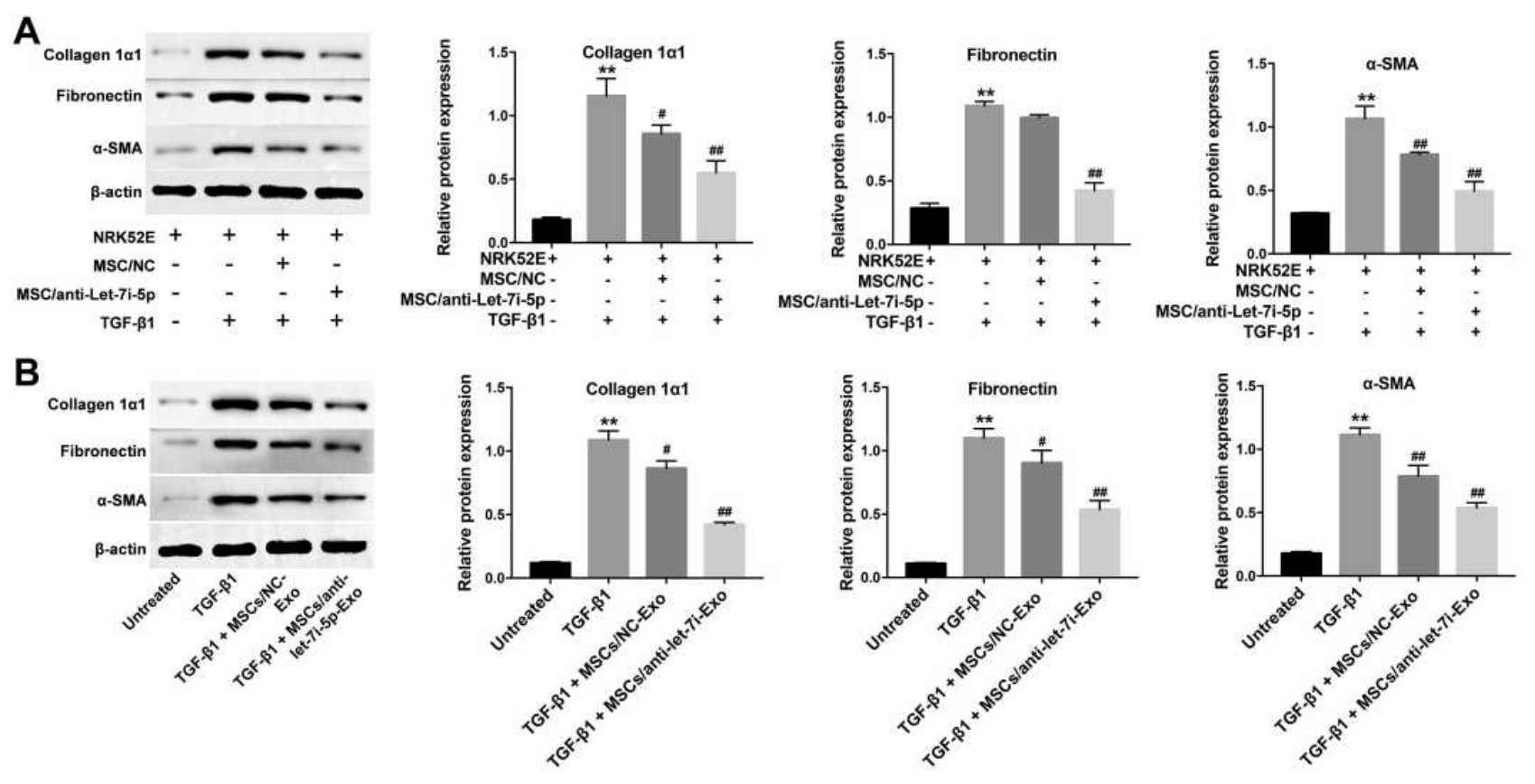

Figure 4 MSCs-secreted anti-let-7i-5p inhibited the ECM deposition and EMT process in TGF- $\beta$ I-treated NRK52E cells. (A) NRK52E cells, with the addition of TGF- $\beta$ I, were indirectly co-cultured with MSCs/anti-let-7i-5p or MSCs/NC. Expressions of collagen I $\alpha$ l, fibronectin and $\alpha$-SMA in NRK52E cells were detected with Western blotting $(n=3)$. The relative expressions of collagen I $\alpha$ I, fibronectin and $\alpha$-SMA were quantified via normalization to $\beta$-actin. $* * P<0.0$ I, compared with NRK52E group; ${ }^{\#} \mathrm{P}<0.05,{ }^{\#} \mathrm{P}<0.01$, compared with the TGF- $\beta$ I group. (B) NRK52E cells, with the addition of TGF- $\beta$ I, were treated with MSCs/anti-let-7i-5p exosomes or MSCs/NC exosomes. Expressions of collagen I $\alpha$ I, fibronectin and $\alpha$-SMA in NRK52E cells were detected with Western blotting ( $n=3$ ). The relative expressions of collagen I $\alpha$, fibronectin and $\alpha$-SMA were quantified via normalization to $\beta$-actin. ${ }^{* * P}<0.0$ l, compared with untreated group; ${ }^{\#} \mathrm{P}<0.05$, ${ }^{\# \#} \mathrm{P}<0.0 \mathrm{I}$, compared with the TGF- $\beta$ I group. The significance between four groups was analyzed by one-way ANOVA.

collagen deposition in the kidneys of UUO mice (Figure 7C). Meanwhile, the extent of fibrosis was significantly decreased in the kidneys of UUO mice that received MSCs/anti-let-7i-5p exosomes compared to UUO group (Figure 7D). Furthermore, the results of IHC analysis showed that the expressions of collagen $1 \alpha 1$, fibronectin and $\alpha$-SMA were markedly increased in the kidneys of UUO mice; whereas these changes were reversed in UUO mice that received MSCs/NC exosomes or MSCs/ anti-let-7i-5p exosomes (Figure 8A and B). These results suggested that MSCs/anti-let-7i-5p exosomes could alleviate renal fibrosis in UUO mice.

\section{MSCs/Anti-Let-7i-5p Exosomes}

\section{Alleviated Renal Fibrosis in UUO Mice via Upregulation of TSCI}

In order to investigate the anti-fibrotic mechanism of MSCs/anti-let-7i-5p exosomes in vivo, TSC1/mTOR signaling pathway was examined. As shown in Figure 9A and B, exosomes-mediated delivery of anti-let-7i-5p significantly upregulated the expression of TSC1 and downregulated mTORC1, p70S6K and 4E-BP1 phosphorylation in the kidneys of UUO mice. In addition, MSCs/anti-let-7i$5 p$ exosomes notably decreased the level of let-7i-5p in the kidneys of mice subjected to UUO (Figure 9C). To sum up, MSCs/anti-let-7i-5p exosomes could alleviate renal fibrosis in UUO mice via upregulation of TSC1.

\section{Discussion}

In this study, we found that the level of let-7i-5p was markedly upregulated in TGF- $\beta 1$-stimulated NRK52E cells and in the kidneys following UUO. Moreover, exosomes-mediated delivery of anti-let-7i-5p exerts antifibrotic effects in TGF- $\beta 1$-induced fibrogenic responses in NRK52E cells in vitro as well as in UUO-induced renal fibrosis model in vivo. Our findings establish an important link between exosomal let-7i-5p and renal fibrosis.

Evidences have shown that MSCs-Exo could attenuate the progression of renal fibrosis in CKD. ${ }^{41,42}$ Liu et al found that human umbilical cord MSCs-derived exosomes could attenuate UUO-induced renal fibrosis. ${ }^{41}$ In this study, we found that MSCs/NC-Exo improved renal function and attenuated renal fibrosis in mice subjected to UUO, which was consistent with the previous study. In 

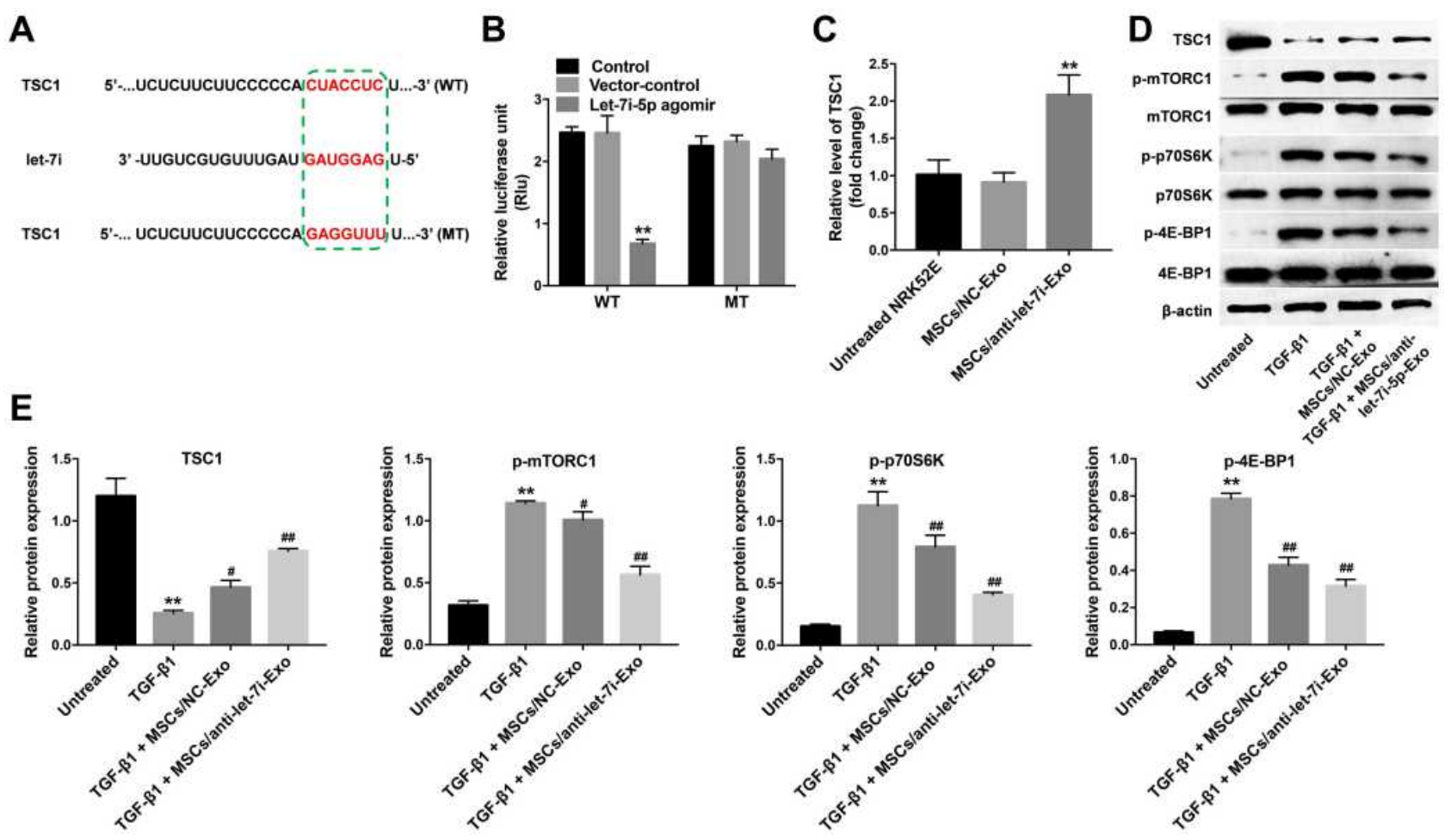

Figure 5 TSCI is a functional target of let-7i-5p in NRK52E cells. (A) Schematic diagram of binding sites between let-7i-5p and TSCI, as well as the mutation of binding sites in TSCI. (B) Luciferase assay of cells transfected with TSCI-wild type (WT) or TSCI-mutant (MT) reporter together with let-7i-5p or NC. **P < 0.01 , compared with vector-control group. (C) NRK52E cells were treated with MSCs/anti-let-7i-5p exosomes or MSCs/NC exosomes. RT-qPCR was used to detect the level of TSCI in cells (n = 3). ${ }^{* * P}<0.0 \mathrm{I}$, compared with MSCs/NC-Exo group. (D and E) NRK52E cells, with the addition of TGF- $\beta$ I, were treated with MSCs/anti-let-7i-5p exosomes or MSCs/NC exosomes. Expressions of TSCI, p-mTORCI, mTORCI, p-p70S6K, p70S6K, p-4E-BPI and 4E-BPI in NRK52E cells were detected with Western blotting ( $=3$ ). The relative expressions of TSCI, p-mTORCI, p-p70S6K and p-4E-BPI were quantified via normalization to $\beta$-actin, mTORCI, p70S6K and 4E-BPI. **P < $0.0 \mathrm{I}$, compared with untreated group; ${ }^{\#} \mathrm{P}<0.05,{ }^{\#} \mathrm{P}<0.0 \mathrm{I}$, compared with the TGF- $\beta$ I group. The significance between three or more groups was analyzed by one-way ANOVA.

addition, miRNA can be transferred between MSCs and injured epithelial cells via exosomes. ${ }^{43}$ For example, exosomal miR-122 derived from MSCs can be delivered to hepatic stellate cells, consequently inhibits the activation of hepatic stellate cells, alleviates collagen deposition and alleviates liver fibrosis. ${ }^{44}$ Exosome-mediated miR-let-7c transferred from MSCs to the kidneys of UUO mice inhibited TGF- $\beta$ R 1 gene expression and attenuated renal fibrosis in vivo. ${ }^{29}$ These results indicated that exosomemediated transfer of miRNA between MSCs and injured epithelial cells is important for the progression of renal fibrosis. We reported that anti-let-7i-5p oligonucleotides can be transferred from MSCs to injured kidney tubular epithelial cells as well as the obstructed kidneys via exosomes. In addition, exosomal anti-let-7i-5p from MSCs significantly decreased the level of let-7i-5p in TGF$\beta 1$-stimulated NRK52E cells and in the kidneys following UUO. Collectively, MSCs-Exo can deliver the anti-let-7i$5 p$ to injured renal cells, causing inhibition of let-7i-5p.

EMT plays important roles in the development of renal fibrosis. ${ }^{45}$ In this process, renal tubular epithelial cells lost some epithelial markers while obtaining partial mesenchymal markers, including $\alpha$-SMA, resulting in a significant increase on the number of myofibroblasts, and eventually leading to renal fibrosis. ${ }^{46}$ Meanwhile, EMT can promote renal fibrosis through increasing deposition of ECM protein, such as collagen $1 \alpha 1$, fibronectin. ${ }^{47,48}$ TGF- $\beta 1$ induced EMT process, leading to excessive production and deposition of ECM proteins in the renal interstitium. ${ }^{49}$ In this study, we found that MSCs/anti-let $-7 \mathrm{i}-5 \mathrm{p}$ exosomes suppressed TGF- $\beta 1$-induced myofibroblast formation and ECM production in NRK52E cells. In addition, MSCs/anti-let-7i-5p exosomes also attenuated renal fibrosis in obstructed kidneys, as evidenced by decreased levels of collagen $1 \alpha 1$, fibronectin and $\alpha$-SMA. Meanwhile, MSCs/anti-let-7i-5p exosomes inhibited UUO-induced increase of BUN and CR levels, indicating that exosomal anti-miR-let-7 can improve UUO-induced renal dysfunction. These results suggested that exosomal anti-miR-let-7 provided reno-protective effects via attenuating renal fibrosis in vitro and in vivo. 


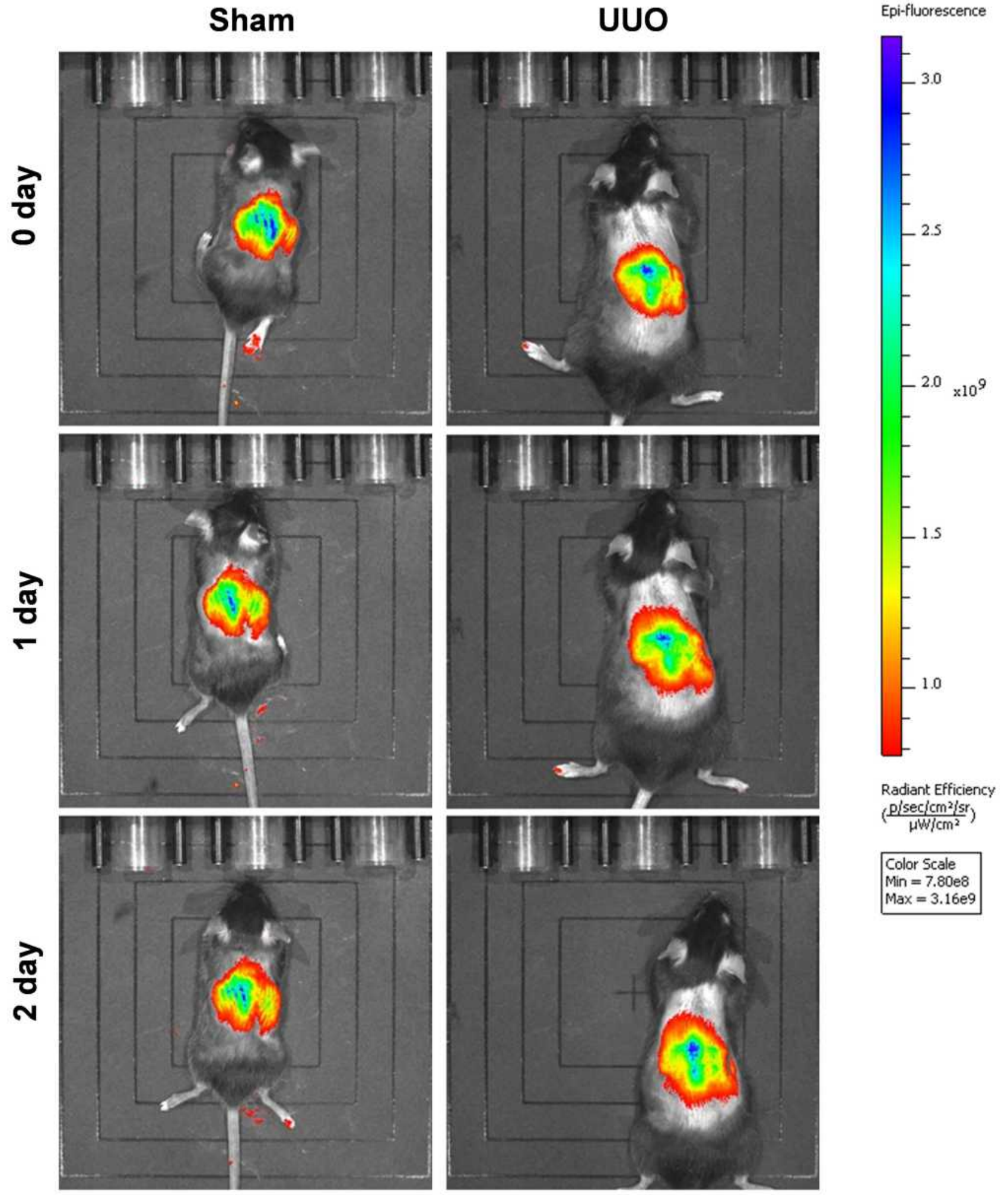

Figure 6 In vivo distribution of MSCs/Exo. In vivo fluorescent images of mice with sham-operated controls or UUO injury after intravenous injection of PKH67 labeled MSCs-Exo at different time point.

Let-7i-5p has been shown to be participate in cell proliferation and migration. ${ }^{50}$ However, the role of let-7i$5 p$ in the development of renal fibrosis has not been illustrated. Evidence shown that miRNAs exert their functions predominantly based on their target genes..$^{51}$ Our data found that TSC1 was a binding target of let-7i-5p. TSC1 is 

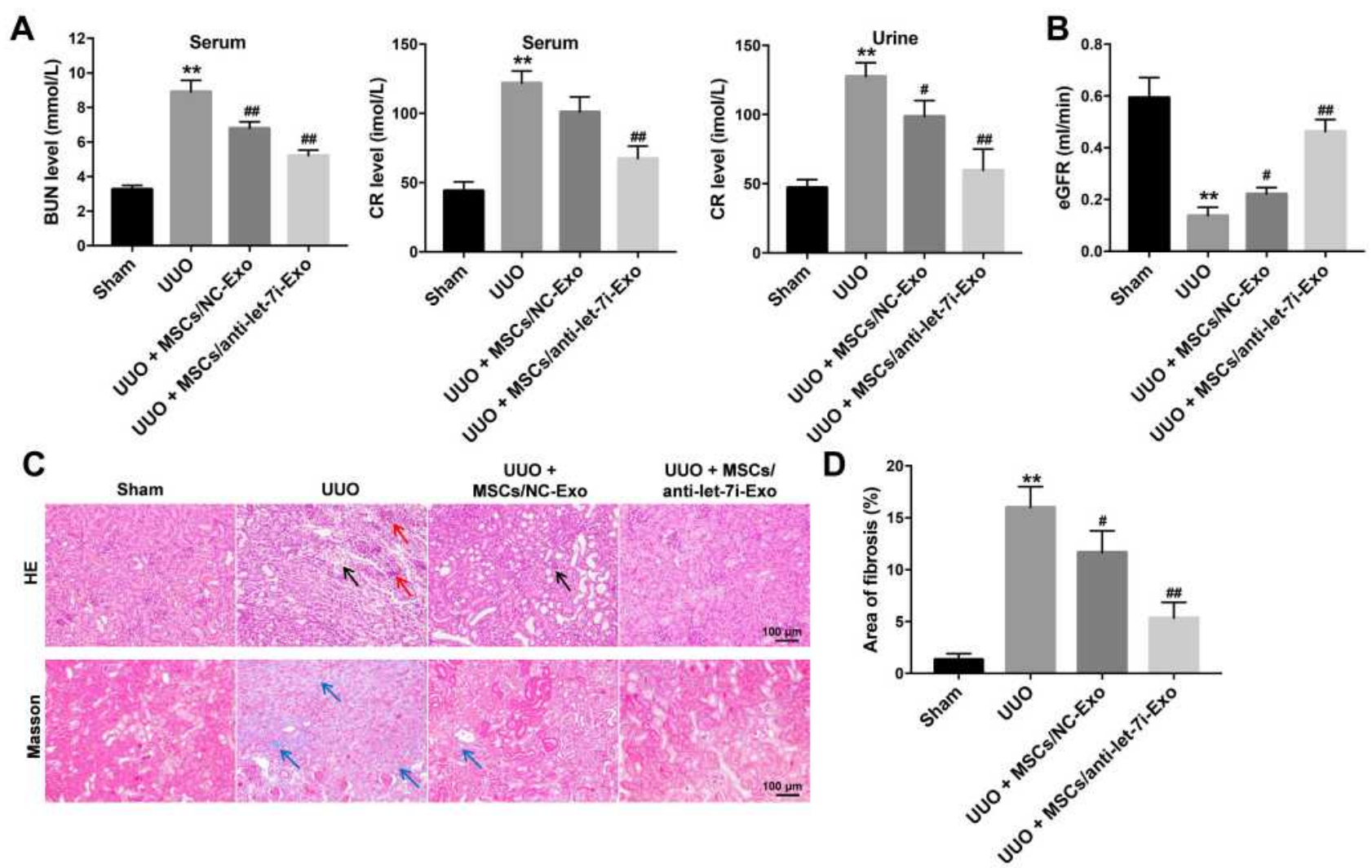

Figure 7 MSCs/anti-let-7i-5p exosomes alleviated renal fibrosis in UUO mice. (A) ELISA assay was used to measure the levels of BUN and CR in serum of mice and the level of CR in urine of mice. (B) Measurement of eGFR in mice. (C) H\&E and Masson's trichrome staining assay was used to analyze renal fibrosis and collagen deposition (magnification, $\times 200$ ). Red arrows, glomerular injury; black arrows, renal interstitial edema; blue arrow, collagen fibers. (D) Total renal fibrotic area was measured by ImagePro Plus. ${ }^{* * P}<0.01$, compared with Sham group; ${ }^{\#} \mathrm{P}<0.05,{ }^{\# \#} \mathrm{P}<0.01$, compared with the UUO group. The significance between four groups was analyzed by one-way ANOVA.
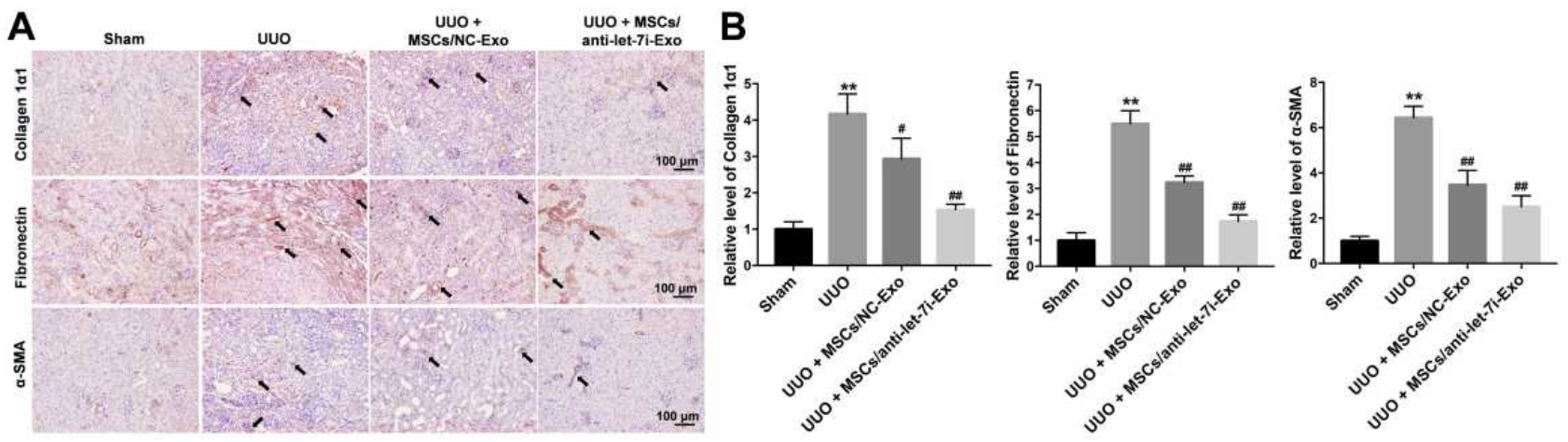

Figure $8 \mathrm{MSC}$ /anti-let-7i-5p exosomes decreased the expressions of EMT-related proteins in UUO mice. (A and B) IHC staining of collagen I $\alpha$ I, fibronectin and $\alpha$-SMA (indicated by arrowheads) in the kidneys of mice (magnification, $\times 200 ; n=3$ ). ${ }^{* *} \mathrm{P}<0.0 \mathrm{I}$, compared with Sham group; ${ }^{\#} \mathrm{P}<0.05$, ${ }^{\# \#} \mathrm{P}<0.0 \mathrm{I}$, compared with the UUO group. The significance between four groups was analyzed by one-way ANOVA.

an upstream negative regulator of mTOR signaling. ${ }^{52}$ Shao et al found that miR-29c attenuated renal fibrosis in vitro and in vivo via inhibition of mTOR signaling. ${ }^{53}$ Our results show that exosomal anti-miR-let-7 increased TSC1 expression level in the damaged kidneys and subsequently reduced the phosphorylation of its downstream targets mTOR, p70S6K and 4E-BP1. In support of our current findings, $\mathrm{Lu}$ et al found that overexpression of TSC1 attenuated renal fibrosis in diabetic nephropathy via inactivation of mTOR signaling. ${ }^{54}$ Our data suggest that MSCs-secreted exosomal let-7i-5p attenuated renal fibrosis by initiating the TSC $1 / \mathrm{mTOR}$ pathway. 

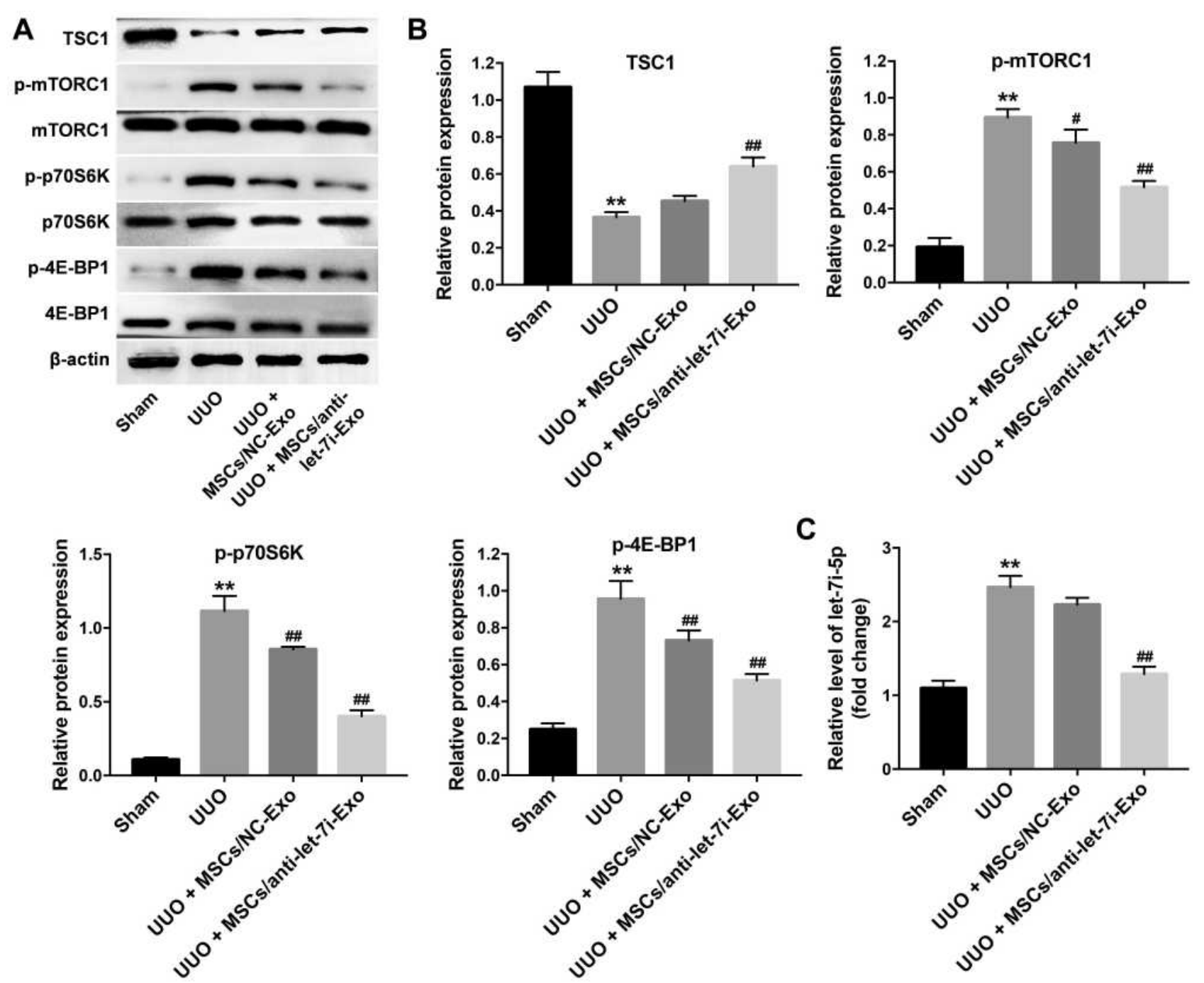

Figure $9 \mathrm{MSC}$ /anti-let-7i-5p exosomes alleviated renal fibrosis in UUO mice via upregulation of TSCI. (A) Expressions of TSCI, p-mTORCI, mTORCl, p-p70S6K, p70S6K, p-4E-BPI and 4E-BPI in the kidneys of mice were detected with Western blotting $(n=3)$. (B) The relative expressions of TSCI, p-mTORCI, p-p70S6K and p-4EBPI were quantified via normalization to $\beta$-actin, mTORCI, p70S6K and 4E-BPI. (C) RT-qPCR was used to analyze the level of let-7i-5p in the kidneys of mice $(n=3)$. *** $<$ 0.01 , compared with Sham group; ${ }^{\#} \mathrm{P}<0.05,{ }^{\# P}<0.01$, compared with the UUO group. The significance between four groups was analyzed by one-way ANOVA.

\section{Conclusion}

In this study, we identified a role of let-7i-5p as a key regulator of TGF- $\beta$-induced fibrogenesis in vitro and UUO-induced renal fibrosis model in vivo. In addition, exosomal anti-let-7i-5p from MSCs exerts anti-fibrotic effects in TGF- $\beta 1$-induced fibrogenic responses in NRK52E cells in vitro as well as in UUO-induced renal fibrosis model in vivo via activating the TSC1/mTOR pathway. These results provided a novel perspective on improving renal fibrosis by MSCs-Exo.

\section{Ethics Approval and Consent to Participate}

All animal procedures were approved by the Institutional Ethical Committee of the Zhejiang Provincial People's Hospital. National Institutes of Health Guide for the Care and Use of Laboratory Animals was followed strictly.

\section{Funding}

The Construction of Key Projects by Zhejiang Provincial Ministry (Project No. WKJ-ZJ-1915, WKJ-ZJ-2017). The 
Zhejiang Province Chinese Medicine Modernization Program (Project No. 2020ZX001). The General Project of Zhejiang Education Department (Project No. Y201942823).

\section{Disclosure}

Juan Jin and Fengmei Qian should be considered as cofirst authors. The authors declare no competing financial interests.

\section{References}

1. Chevalier RL. Evolution, kidney development, and chronic kidney disease. Semin Cell Dev Biol. 2019;91:119-131. doi:10.1016/j. semcdb.2018.05.024

2. Parrish AR. Advances in chronic kidney disease. Int $J$ Mol Sci. 2016;17(8):1314. doi:10.3390/ijms17081314

3. Chen RA, Scott S, Mattern WD, Mohini R, Nissenson AR. The case for disease management in chronic kidney disease. Dis Manag. 2006;9(2):86-92. doi:10.1089/dis.2006.9.86

4. Mallipattu SK, He JC. The beneficial role of retinoids in glomerular disease. Front Med (Lausanne). 2015;2:16. doi:10.3389/ fmed.2015.00016

5. Floege J, Barbour SJ, Cattran DC, et al. Management and treatment of glomerular diseases (part 1): conclusions from a kidney disease: improving global outcomes (KDIGO) controversies conference. Kidney Int. 2019;95(2):268-280. doi:10.1016/j.kint.2018.10.018

6. Gaitonde DY, Cook DL, Rivera IM. Chronic kidney disease: detection and evaluation. Am Fam Physician. 2017;96(12):776-783.

7. Wouters OJ, O'Donoghue DJ, Ritchie J, Kanavos PG, Narva AS. Early chronic kidney disease: diagnosis, management and models of care. Nat Rev Nephrol. 2015;11(8):491-502. doi:10.1038/ nrneph.2015.85

8. Ammirati AL. Chronic kidney disease. Rev Assoc Med Bras. 2020;66 (Suppl 1):s03-s09. doi:10.1590/1806-9282.66.s1.3

9. Hsieh CY, Lai CC, Lee JS, Tseng CC. Subdural hemorrhage in patients with end-stage renal disease requiring dialysis: a Single-Center Study. Acta Neurol Taiwan. 2018;27(1):9-17.

10. Giatras I, Lau J, Levey AS. Effect of angiotensin-converting enzyme inhibitors on the progression of nondiabetic renal disease: a meta-analysis of randomized trials. angiotensin-converting-enzyme inhibition and progressive renal disease study group. Ann Intern Med. 1997;127(5):337-345. doi:10.7326/0003-4819-1275-199709010-00001

11. Pereira BJ. Optimization of pre-ESRD care: the key to improved dialysis outcomes. Kidney Int. 2000;57(1):351-365. doi:10.1046/ j.1523-1755.2000.00840.x

12. Lv W, Fan F, Wang Y, et al. Therapeutic potential of microRNAs for the treatment of renal fibrosis and CKD. Physiol Genomics. 2018;50 (1):20-34. doi:10.1152/physiolgenomics.00039.2017

13. Meng XM, Tang PM, Li J, Lan HY. TGF- $\beta /$ Smad signaling in renal fibrosis. Front Physiol. 2015;6:82. doi:10.3389/fphys.2015.00082

14. Solez K, Axelsen RA, Benediktsson H, et al. International standardization of criteria for the histologic diagnosis of renal allograft rejection: the Banff working classification of kidney transplant pathology. Kidney Int. 1993;44(2):411-422. doi:10.1038/ki.1993.259

15. Farris AB, Adams CD, Brousaides N, et al. Morphometric and visual evaluation of fibrosis in renal biopsies. J Am Soc Nephrol. 2011;22 (1):176-186. doi:10.1681/ASN.2009091005

16. Chou YH, Chu TS, Lin SL. Role of renin-angiotensin system in acute kidney injury-chronic kidney disease transition. Nephrology (Carlton). 2018;23(Suppl 4):121-125. doi:10.1111/nep.13467
17. Tu Y, Gu L, Chen D, et al. Rhein inhibits autophagy in rat renal tubular cells by regulation of AMPK/mTOR signaling. Sci Rep. 2017;7(1):43790. doi:10.1038/srep43790

18. Xu Y, Ling Y, Yang F, et al. The mTOR/p70S6K1 signaling pathway in renal fibrosis of children with immunoglobulin A nephropathy. $J \quad$ Renin Angiotensin Aldosterone Syst. 2017;18 (3):1470320317717831. doi:10.1177/1470320317717831

19. Dou F, Liu Y, Liu L, et al. Aloe-emodin ameliorates renal fibrosis via inhibiting PI3K/Akt/mTOR signaling pathway in vivo and in vitro. Rejuvenation Res. 2019;22(3):218-229. doi:10.1089/rej.2018.2104

20. Eirin A, Zhu XY, Puranik AS, et al. Mesenchymal stem cell-derived extracellular vesicles attenuate kidney inflammation. Kidney Int. 2017;92(1):114-124. doi:10.1016/j.kint.2016.12.023

21. Zhuang Q, Ma R, Yin Y, et al. Mesenchymal stem cells in renal fibrosis: the flame of cytotherapy. Stem Cells Int. 2019;2019:8387350. doi:10.1155/2019/8387350

22. Păunescu V, Deak E, Herman D, et al. In vitro differentiation of human mesenchymal stem cells to epithelial lineage. $J$ Cell Mol Med. 2007;11(3):502-508. doi:10.1111/j.1582-4934.2007.00041.x

23. Singaravelu K, Padanilam BJ. In vitro differentiation of MSC into cells with a renal tubular epithelial-like phenotype. Ren Fail. 2009;31 (6):492-502. doi:10.1080/08860220902928981

24. Gnecchi M, Danieli P, Malpasso G, Ciuffreda MC. Paracrine mechanisms of mesenchymal stem cells in tissue repair. Methods Mol Biol. 2016;1416:123-146.

25. Zhu F, Chong lee Shin OLS, Pei G, et al. Adipose-derived mesenchymal stem cells employed exosomes to attenuate AKI-CKD transition through tubular epithelial cell dependent Sox9 activation. Oncotarget. 2017;8(41):70707-70726. doi:10.18632/oncotarget.19979

26. Lou G, Chen Z, Zheng M, Liu Y. Mesenchymal stem cell-derived exosomes as a new therapeutic strategy for liver diseases. Exp Mol Med. 2017;49(6):e346. doi:10.1038/emm.2017.63

27. Dai J, Su Y, Zhong S, et al. Exosomes: key players in cancer and potential therapeutic strategy. Signal Transduct Target Ther. 2020;5 (1):145. doi:10.1038/s41392-020-00261-0

28. Wang Y, Guo YF, Fu GP, et al. Protective effect of miRNA-containing extracellular vesicles derived from mesenchymal stromal cells of old rats on renal function in chronic kidney disease. Stem Cell Res Ther. 2020;11(1):274. doi:10.1186/s13287-020-01792-7

29. Wang B, Yao K, Huuskes BM, et al. Mesenchymal stem cells deliver exogenous MicroRNA-let7c via exosomes to attenuate renal fibrosis. Mol Ther. 2016;24(7):1290-1301. doi:10.1038/mt.2016.90

30. Conserva F, Barozzino M, Pesce F, et al. Urinary miRNA-27b-3p and miRNA-1228-3p correlate with the progression of kidney fibrosis in diabetic nephropathy. Sci Rep. 2019;9(1):11357. doi:10.1038/s41598019-47778-1

31. Zhou H, Hasni SA, Perez P, et al. miR-150 promotes renal fibrosis in lupus nephritis by downregulating SOCS1. J Am Soc Nephrol. 2013;24(7):1073-1087. doi:10.1681/ASN.2012080849

32. Liu Y, Bi X, Xiong J, et al. MicroRNA-34a promotes renal fibrosis by downregulation of klotho in tubular epithelial cells. Mol Ther. 2019;27(5):1051-1065. doi:10.1016/j.ymthe.2019.02.009

33. Grgic I, Kiss E, Kaistha BP, et al. Renal fibrosis is attenuated by targeted disruption of KCa3.1 potassium channels. Proc Natl Acad Sci U S A. 2009;106(34):14518-14523. doi:10.1073/ pnas.0903458106

34. Kim DH, Kim H, Choi YJ, et al. Exosomal PD-L1 promotes tumor growth through immune escape in non-small cell lung cancer. Exp Mol Med. 2019;51(8):1-13.

35. Zhou X, Li Z, Qi M, et al. Brown adipose tissue-derived exosomes mitigate the metabolic syndrome in high fat diet mice. Theranostics. 2020;10(18):8197-8210. doi:10.7150/thno.43968

36. Li W, Ding Y, Smedley C, et al. Increased glomerular filtration rate and impaired contractile function of mesangial cells in TRPC6 knockout mice. Sci Rep. 2017;7(1):4145. doi:10.1038/s41598-01704067-z 
37. Gooch JL, King C, Francis CE, Garcia PS, Bai Y. Cyclosporine A alters expression of renal microRNAs: new insights into calcineurin inhibitor nephrotoxicity. PLoS One. 2017;12(4):e0175242. doi:10.1371/journal.pone.0175242

38. Luan J, Fu J, Chen C, et al. LNA-anti-miR-150 ameliorated kidney injury of lupus nephritis by inhibiting renal fibrosis and macrophage infiltration. Arthritis Res Ther. 2019;21(1):276. doi:10.1186/s13075019-2044-2

39. Chen L, Wang Y, Li S, et al. Exosomes derived from GDNF-modified human adipose mesenchymal stem cells ameliorate peritubular capillary loss in tubulointerstitial fibrosis by activating the SIRT1/eNOS signaling pathway. Theranostics. 2020;10(20):9425-9442. doi: $10.7150 /$ thno. 43315

40. Nagaishi K, Mizue Y, Chikenji T, et al. Mesenchymal stem cell therapy ameliorates diabetic nephropathy via the paracrine effect of renal trophic factors including exosomes. Sci Rep. 2016;6(1):34842. doi:10.1038/srep34842

41. Liu B, Hu D, Zhou Y, et al. Exosomes released by human umbilical cord mesenchymal stem cells protect against renal interstitial fibrosis through ROS-mediated P38MAPK/ERK signaling pathway. Am $J$ Transl Res. 2020;12(9):4998-5014.

42. Xiang E, Han B, Zhang Q, et al. Human umbilical cord-derived mesenchymal stem cells prevent the progression of early diabetic nephropathy through inhibiting inflammation and fibrosis. Stem Cell Res Ther. 2020;11(1):336. doi:10.1186/s13287-020-01852-y

43. Qiu G, Zheng G, Ge M, et al. Mesenchymal stem cell-derived extracellular vesicles affect disease outcomes via transfer of microRNAs. Stem Cell Res Ther. 2018;9(1):320. doi:10.1186/s13287-018-1069-9

44. Lou G, Yang Y, Liu F, et al. MiR-122 modification enhances the therapeutic efficacy of adipose tissue-derived mesenchymal stem cells against liver fibrosis. $J$ Cell Mol Med. 2017;21 (11):2963-2973. doi:10.1111/jcmm.13208

45. Zhou T, Luo M, Cai W, et al. Runt-related transcription factor 1 (RUNX1) promotes TGF- $\beta$-induced renal tubular epithelial-tomesenchymal transition (EMT) and renal fibrosis through the PI3K subunit p1108. EBioMedicine. 2018;31:217-225. doi:10.1016/j. ebiom.2018.04.023
46. Liu B, Ding F, Hu D, et al. Human umbilical cord mesenchymal stem cell conditioned medium attenuates renal fibrosis by reducing inflammation and epithelial-to-mesenchymal transition via the TLR4/NF$\kappa \mathrm{B}$ signaling pathway in vivo and in vitro. Stem Cell Res Ther. 2018;9(1):7. doi:10.1186/s13287-017-0760-6

47. Zhang HJ, Zhang YN, Zhou H, et al. IL-17A promotes initiation and development of intestinal fibrosis through EMT. Dig Dis Sci. 2018;63 (11):2898-2909. doi:10.1007/s10620-018-5234-x

48. Ma B, Yang L, Jing R, et al. Effects of Interleukin-6 on posterior capsular opacification. Exp Eye Res. 2018;172:94-103. doi:10.1016/j. exer.2018.03.013

49. Zeisberg M, Kalluri R. The role of epithelial-to-mesenchymal transition in renal fibrosis. $J$ Mol Med (Berl). 2004;82(3):175-181. doi:10.1007/s00109-003-0517-9

50. Sun X, Xue H, Xiong Y, et al. GALE promotes the proliferation and migration of glioblastoma cells and is regulated by miR-let-7i-5p. Cancer Manag Res. 2019;11:10539-10554. doi:10.2147/CMAR. $\mathrm{S} 221585$

51. Jin HY, Oda H, Chen $P$, et al. Differential sensitivity of target genes to translational repression by miR-17 92. PLoS Genet. 2017;13(2): e1006623. doi:10.1371/journal.pgen.1006623

52. Shi L, Chen X, Zang A, et al. TSC1/mTOR-controlled metabolic-epigenetic cross talk underpins DC control of CD8+ T-cell homeostasis. PLoS Biol. 2019;17(8):e3000420. doi:10.1371/ journal.pbio. 3000420

53. Shao H, Huang Y, Hu HL, Fan WX, Yin XN. Effect of miR-29c on renal fibrosis in diabetic rats via the AMPK/mTOR signaling pathway. Eur Rev Med Pharmacol Sci. 2019;23(14):6250-6256. doi:10.26355/eurrev_201907_18445

54. Lu Q, Chen YB, Yang $\mathrm{H}$, et al. Inactivation of $\mathrm{TSC} 1$ promotes epithelial-mesenchymal transition of renal tubular epithelial cells in mouse diabetic nephropathy. Acta Pharmacol Sin. 2019;40 (12):1555-1567. doi:10.1038/s41401-019-0244-6
International Journal of Nanomedicine

\section{Publish your work in this journal}

The International Journal of Nanomedicine is an international, peerreviewed journal focusing on the application of nanotechnology in diagnostics, therapeutics, and drug delivery systems throughout the biomedical field. This journal is indexed on PubMed Central, MedLine, CAS, SciSearch ${ }^{\mathbb{}}$, Current Contents ${ }^{\mathbb{R}} /$ Clinical Medicine,
Journal Citation Reports/Science Edition, EMBase, Scopus and the Elsevier Bibliographic databases. The manuscript management system is completely online and includes a very quick and fair peer-review system, which is all easy to use. Visit http://www.dovepress.com/ testimonials.php to read real quotes from published authors. 